UNIVERSIDADE DE SÃO PAULO

FACULDADE DE ECONOMIA, ADMINISTRAÇÃO E CONTABILIDADE DEPARTAMENTO DE ECONOMIA PROGRAMA DE PÓS-GRADUAÇÃO EM ECONOMIA

OS ASPECTOS ECONÔMICOS DA DISCRIMINAÇÃO RACIAL NO BRASIL Gustavo Andrey de Almeida Lopes Fernandes

Orientador: Prof. Dr. Denisard Cnéio de Oliveira Alves 
Prof. Dr. João Grandino Rodas Reitor da Universidade de São Paulo

Prof. Dr. Reinaldo Guerreiro

Diretor da Faculdade de Economia, Administração e Contabilidade

Profa.Dra. Elizabeth Maria Mercier Querido Farina

Chefe do Departamento de Economia

Prof. Dr. Pedro Garcia Duarte

Coordenador do Programa de Pós-Graduação em Economia 
GUSTAVO ANDREY DE ALMEIDA LOPES FERNANDES

\section{OS ASPECTOS ECONÔMICOS DA DISCRIMINAÇÃO RACIAL NO BRASIL}

Tese apresentada ao Departamento de Economia da Faculdade de Economia, Administração e Contabilidade da Universidade de São Paulo como requisito para a obtenção do título de Doutor em Ciências.

Orientador: Prof. Dr. Denisard Cnéio de Oliveira Alves

\section{Versão Corrigida}

(versão original disponível na Faculdade de Economia, Administração e Contabilidade)

SÃO PAULO 


\section{FICHA CATALOGRÁFICA}

Elaborada pela Seção de Processamento Técnico do SBD/FEA/USP

Fernandes, Gustavo Andrey de Almeida Lopes

Os aspectos econômicos da discriminação racial no Brasil / Gustavo Andrey de Almeida Lopes Fernandes. -- São Paulo, 2011.

$$
188 \mathrm{p} \text {. }
$$

Tese (Doutorado) - Universidade de São Paulo, 2012.

Orientador: Denisard Cnéio de Oliveira Alves.

1. Discriminação racial - Aspectos econômicos 2. Economia do trabalho 3. Econometria 4. Economia I. Universidade de São Paulo. Faculdade de Economia, Administração e Contabilidade II. Título. 